\title{
Patterns of talar articulating facets in adult human calcanei from North-East India and their clinical correlation
}

\author{
K. Anjaneyulu1, Chandra Philips², Binod K. Tamang ${ }^{3}$, Arun Kumar ${ }^{4}$ \\ ${ }^{1}$ Department of Anatomy, KMCT Medical College, Calicut, ${ }^{2}$ Department of Anatomy, Mahatma Gandhi Medical College and Research Institute, \\ Pondicherry, ${ }^{3}$ Department of Anatomy, SMIMS, Sikkim, ${ }^{4}$ Department of Anatomy, DSMCH, Perambalur, Tamilnadu
}

\section{A B S T R A C T}

Calcaneus is the largest tarsal bone of the foot and forms the posterior prominence of the heel. The calcaneus articulates with the talus superiorly at the subtalar joint. The subtalar joint is responsible for the inversion and eversion. There are three facets over upper side of talocalcaneal joint: anterior talar facet, middle and posterior. There are considerable variations in the number and arrangement of these facets. A total of 100 dry adult human calcanei were obtained irrespective of age and sex and evaluated for the pattern of talar articulating facets and measurements were made with digital vernier calliper of $0.01 \mathrm{~mm}$ accuracy at anatomy laboratory of Sikkim Manipal Institute of Medical Sciences, Sikkim. The study revealed that type-A was $31 \%$, type-B was $5 \%$, type-C was $62 \%$ and type-D was $2 \%$. In our study type-C was followed by type-A. These morphological variations on the superior surface of the sustentaculum tali may predispose people to joint instability, ligamentous laxity and the development of arthritic changes in the subtalar joint.

Key words: Calcanei, Articular facets, Pattern, Variation, Subtalar joint, Tarsal coalition

\section{INTRODUCTION}

The calcaneus is a weight bearing tarsal bone situated below the talus and extends behind the talus. The dorsal or superior surface has articular facets on the body and the sustentaculum tali by which it articulates with the talus. Normally there are three facets for synovial joints between calcaneus and talus, Anterior, Middle and Posterior. The anterior and the posterior facets are situated on the body and the middle is situated on the sustentaculum tali. There is considerable variation in the number and arrangement of these facets.

Calcaneus is the most frequently fractured tarsal bone with calcaneal fractures accounting for about $60 \%$ of all major tarsal injuries. The majority of fractures are intra-articular with sub talar joint involvement. Although a talocalcaneal coalition may occur at any of the three facets, the majority of the osseous fusions involve the middle facet. Tarsal coalition is a frequent cause of painful flatfoot. ${ }^{1}$

The morphology of the articular facets of the calcaneus has been a subject of interest to anatomists. The articular facets of the sustentaculamtali have a variety of configurations and are functionally important because they influence the subtalar joint stability. In various alignments of foot, such as the talocalcaneal arthritis and coalition, intra articular fractures and congenital dismorphology, flat foot, valgus deformities the size and shape of the bones, the relationships of the talus and calcaneus with each other and other bones of the foot must be considered for the internal and external fixation and surgical procedure. ${ }^{2}$

A thorough knowledge of the anatomical variations of anterior and middle facets, sustentaculumtali and the sulcus 
in between them is essential in order to keep the osteotomy extraarticularly. ${ }^{3}$

Therefore in this study we aimed to analyse the various patterns of talar articulating facets in the calcanei in north eastern population of India.

\section{MATERIALS AND METHODS}

In this study 100 adult dry human calcanei were studied which were collected from anatomy laboratory of Sikkim Manipal Institute of Medical Sciences, Sikkim. The bones thus collected were of unknown sex and age. Any Pathological or damaged bones were excluded from the study. A digital vernier calliper of $0.01 \mathrm{~mm}$ accuracy was used in this study and photos were taken from Sony digital camera of 16.2 mega pixels. The data obtained from the bones were analysed for patterns of talar articulating facets and were compared with different investigators.

The calcanei were grouped into 4 types by using the classification of Bunning and Barnett $(1963,1965)$ and Gupta et al (1977).

The bones were classified into 4 types. Type A, B, C and D.

In Type-A: The calcanei with two articular facets (Anterior, Middle talar articulating facets) for the talar head and it is further divided into 3 sub types.

In A1 - The separation between the facets is less than $2 \mathrm{~mm}$,

In A2 - The separation between the facets is between $2 \mathrm{~mm}$ to $5 \mathrm{~mm}$,

In A3 - The separation between the facets is more than $5 \mathrm{~mm}$.

In Type - B: There is only one single middle facet on the sustentaculumtali, the anterior talar articulating facet is absent.

In Type - C: The calcanei with the anterior and middle talar articulating facets coalescing in to a common articular facet for the talar head. Further divided into two sub types in this $\mathrm{C} 1$ and $\mathrm{C} 2$.

In C1 - Which has constricted facet.

In C2 - Which has unconstricted facet.

In Type - D: The anterior, middle and posterior talar articulating facets coalesce in to a single facet for the talus.

\section{OBSERVATION}

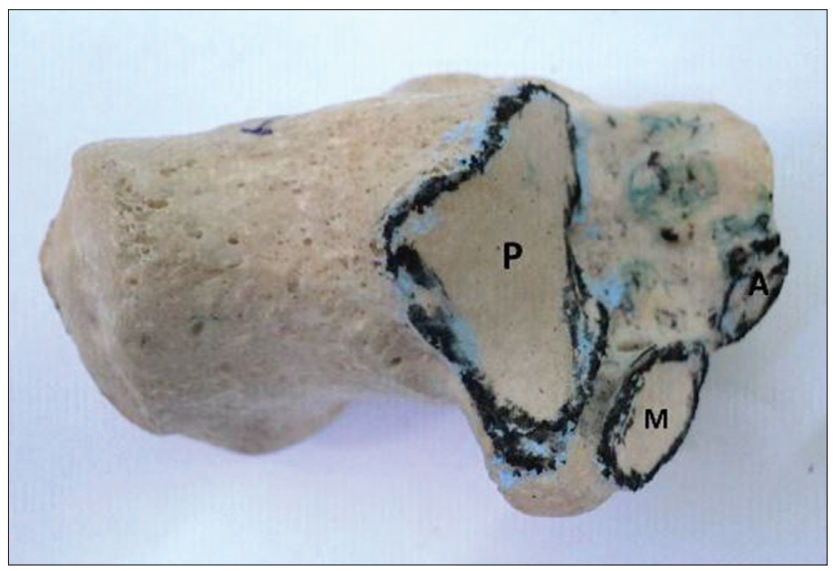

Figure 1: Distance between anterior and middle facets was less than $2 \mathrm{~mm}$

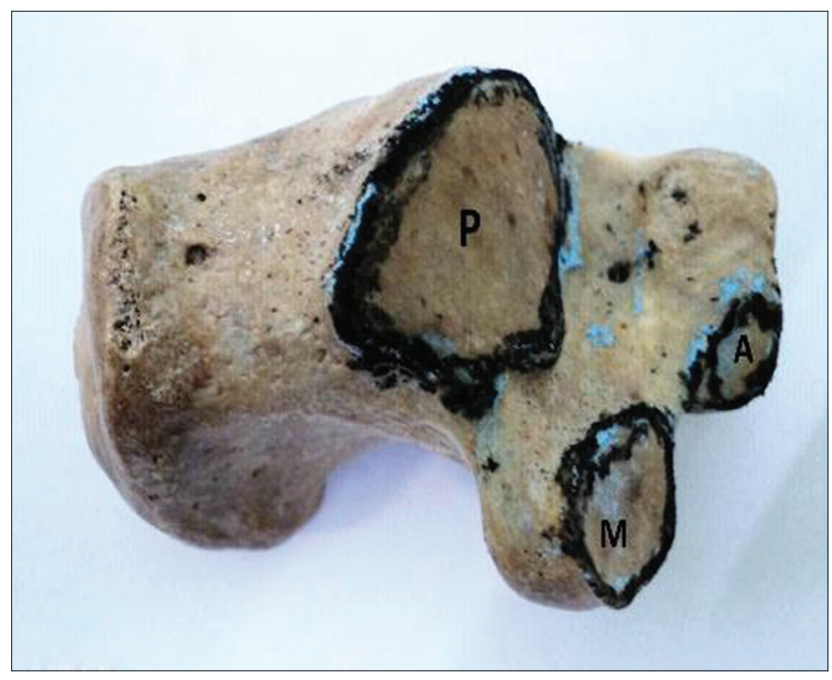

Figure 2: Distance between anterior and middle facets was between 2-5 mm

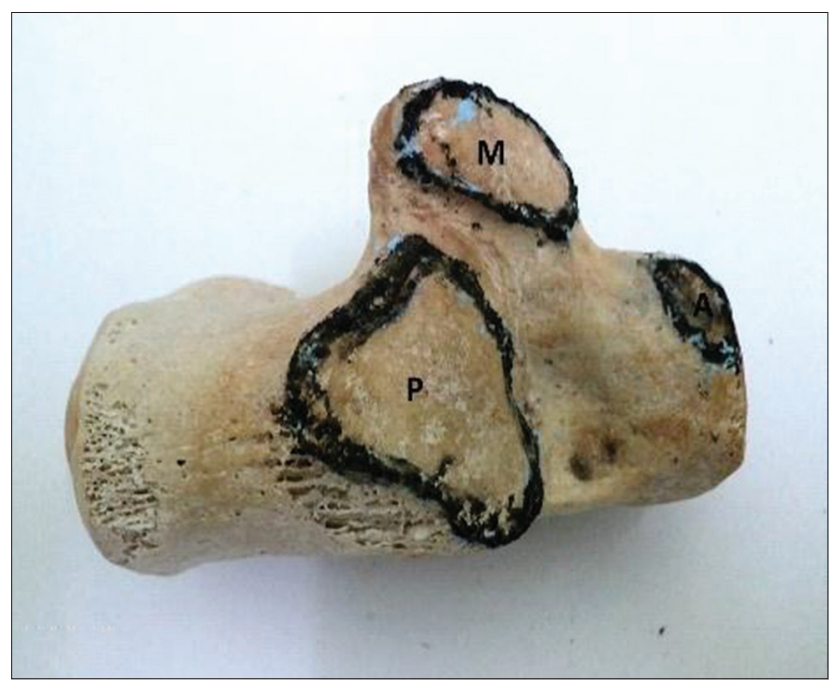

Figure 3: Distance between anterior and middle facets was more than $5 \mathrm{~mm}$. $\mathrm{A}=$ Anterior, $\mathrm{M}=$ Middle, $\mathrm{P}=$ Posterior Facet 


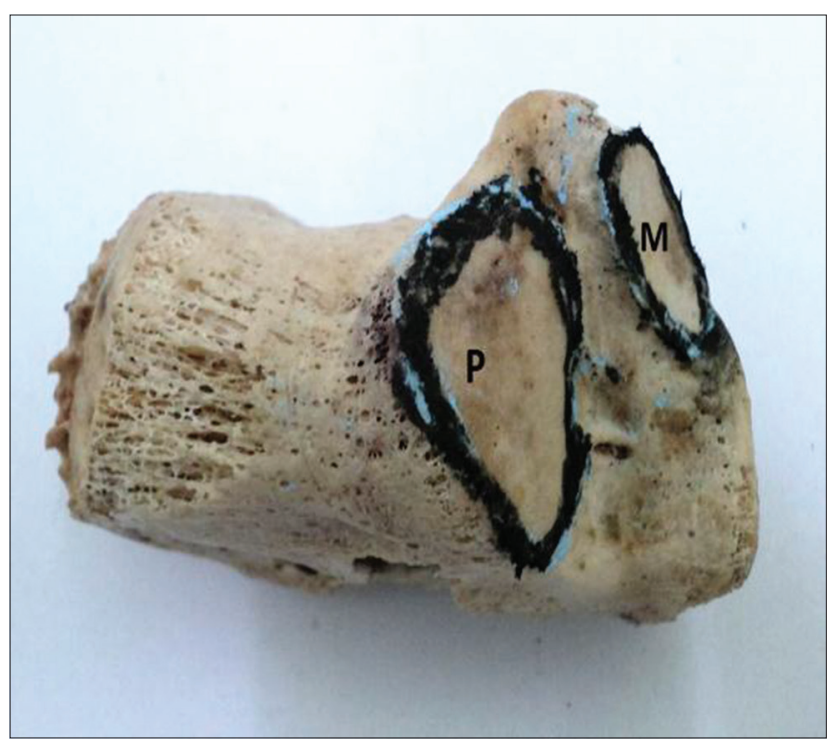

Figure 4: Middle (M), Posterior (P) Facet. This fig shows absence of anterior facet

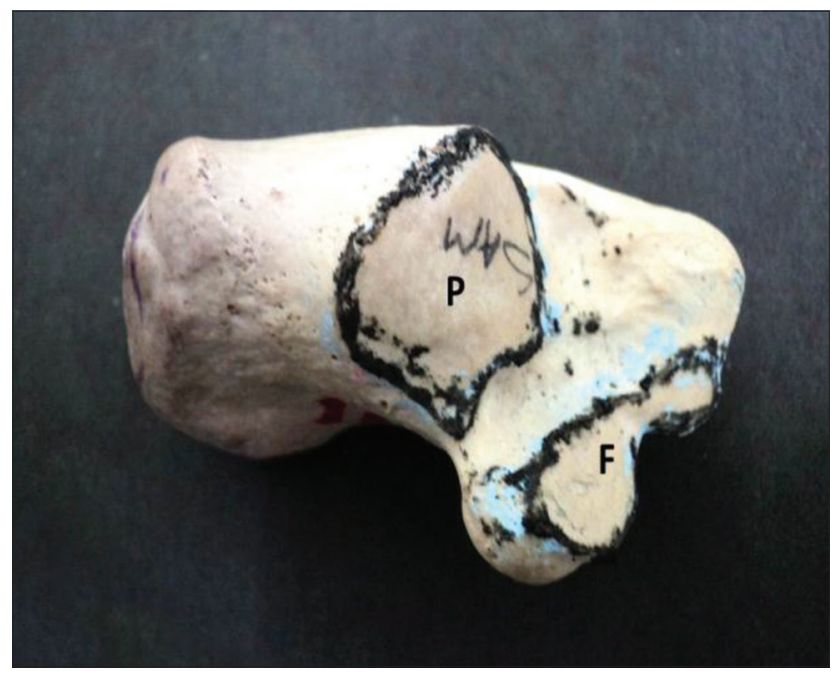

Figure 5: Fused (F), Posterior (P) Facet. Anterior and middle facets are fused and constricted

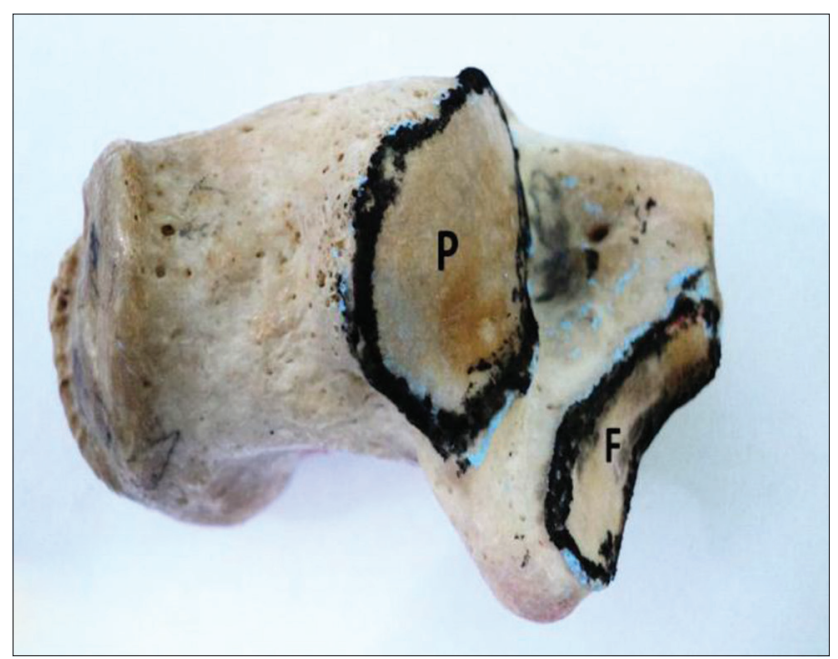

Figure 6: Fused (F), Posterior (P) Facet. Anterior and middle facets are fused and not constricted

Asian Journal of Medical Sciences | Oct-Dec 2014 | Vol 5 | Issue 4

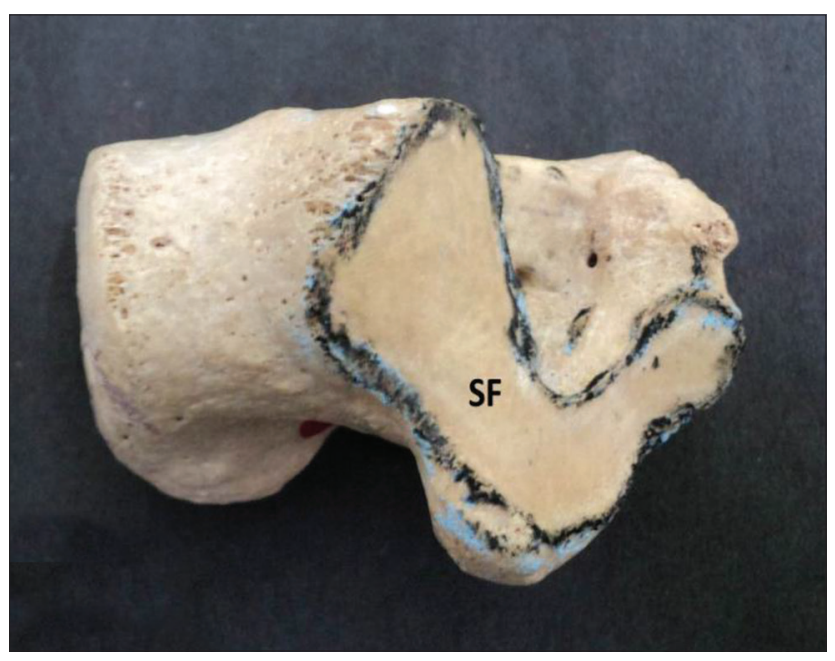

Figure 7: Single Facet (SF). All three facets fused to form single facet

\section{DISCUSSION}

The number and arrangement of the articular facets on the superior surface of the calcaneus designated to carry the head of the talus have been described variously by different authors (Table 1). From this table it is evident that the result of our study is consistent with the studies done previously. In our study we found that the two types, type - C (62\%) and type - A (31\%) constitute the two most common varieties of facets for the articulation with the talus. These findings are consistent with those Indian authors Gupta S.C. et al, ${ }^{4}$ Padmanabhan R. ${ }^{5}$ Bunning and Barnett reported that the incidence of type - $\mathrm{C}$ is significantly higher than the Type - A in both Sudanese and Indian series. ${ }^{6}$ EI-Eishi found type - B to a greater extent in Egyptians which suggests that the facets are genetically determined as mentioned by Bunning and Barnett. ${ }^{7,8}$ Type - D facet is common in Nigerians and Veddah but in a very small sample. Type - D may, therefore perhaps be used as a racial characteristic. ${ }^{9}$ In Campos FF, Pellico LG studies also Type - $\mathrm{C}$ was predominating which was similar to our study. ${ }^{10}$

In all the Indian studies done including the present one, Type - C facets occurred in $62-67 \%$ of calcanei. In western studies also $60-70 \%$ of the calcanei showed the occurrence of Type - C facets. In Egyptian and Spanish, Type - C facets are found in only $50 \%$ of the calcanei, whereas only about $30 \%$ of the European calcanei show this facet.

In Indians and in the most of western populations, the percentage of Type - A facets is just about half of the Type - C facets. On the other hand, in Egyptian and Spanish, the occurrence of Type - C and A facets is approximately same, while in Europeans, Type - A facets are almost twice as common as Type $-\mathrm{C}$ facets. This appears to be an interesting racial characteristic. Many such 


\begin{tabular}{|c|c|c|c|c|c|c|c|c|c|c|}
\hline \multirow[t]{2}{*}{ Author } & \multirow[t]{2}{*}{ Cases } & \multicolumn{9}{|c|}{ Type \% } \\
\hline & & A & A1 & A2 & A3 & B & C & C1 & $\mathrm{C} 2$ & D \\
\hline Laidlaw (1904) & 750 & 32 & & & & & 68 & & & \\
\hline Bunning and Barnett $(1963,1965)$, Veddahs & 10 & & & & & & 60 & & & 40 \\
\hline Bunning and Barnett $(1963,1965)$, Africans & 492 & 36 & & & & & 63 & & & 1 \\
\hline Bunning and Barnett $(1963,1965)$, British & 194 & 67 & & & & & 33 & & & \\
\hline Bunning and Barnett $(1963,1965)$, Indians & 78 & 22 & & & & & 78 & & & \\
\hline El - Eishi (1974), Egyptians & 200 & 40 & & & & 11 & 49 & & & \\
\hline Gupta et al (1977), Indians & 401 & 26 & 9 & 4 & 15 & 5 & 67 & 28 & 39 & 2 \\
\hline Sarrafian (1983) & 50 & 34 & & & & & 64 & & & 2 \\
\hline Campos and Pellico (1989), Spanish & 176 & 42 & 5 & 21 & 16 & 4 & 54 & 29 & 25 & \\
\hline PorntipBoonruangsri (1992), Northeastern Thai & 230 & 40 & 10 & 22 & 8 & 0.57 & 59 & 19 & 40 & 0.43 \\
\hline Francine Drayer - Verhagen (1993), Americans & 191 & 26.7 & & & & 18.85 & 54.4 & & & \\
\hline Saadeh F.A. (2006), Egyptian & 300 & 30.3 & & & & 4.7 & 63 & & & 2 \\
\hline Mujde Uygur (2009), Turkish race & 221 & 39.3 & & & & & 58 & & & \\
\hline IpekErgur (2011) & 80 & 38.7 & & & & & 61.3 & & & \\
\hline Sharada R. (2012), South Indians & 300 & 28.6 & 5.33 & 13.66 & 9.66 & 3 & 67 & 50.3 & 16.6 & 1.33 \\
\hline Present study, North - East Indians & 100 & 31 & 10 & 10 & 11 & 5 & 62 & 43 & 19 & 2 \\
\hline
\end{tabular}

factors as wearing of shoes, squatting habits, genetic and racial differences are the probable reasons for the variations in the facets on the calcanei in the western and Indian series.

Disposition of superior articular facets of the calcaneum is congenital and not a postnatal development, with Type - $\mathrm{C}$ more in females-sexual dimorphism. ${ }^{6}$ Tarsal coalitions are inherited most probably as a unifactorial disorder of autosomal dominant inheritance with full penetrance. ${ }^{11}$ They occur in association with carpal coalitions, phocomelia, hemimelia. ${ }^{2} 83 \%$ of the coalitions are talocalcaneal leading to tarsal tunnel syndrome, osteophyte causing sensory disturbance in the region of medial plantar nerve and restriction of plantar flexion at the ankle joint. ${ }^{12}$ Calcaneal fractures account for $33.3 \%$ of foot fractures, and it was seen that there was 100\% involvement of posterior talocalcaneal facet in intra-articular calcaneal fractures. ${ }^{13}$

Sustentaculumtali facet morphology is an important factor in subtalar joint stability and is consistent with Bruckner's (1987) hypothesis, that the joints with 2-facet configuration are comparatively more stable. Stability of the subtalar joint also depends on the height of the longitudinal arch, which is determined by the inclination of the subtalar joint axis (Perry, 1983; Mann, 1991). A high arch represents a more stable structure and is commonly referred to as a 'rigid' or cavus foot (Norkin, 1983; Glancy, 1984; Bruckner, 1987). ${ }^{14}$ The vastly increased pressure on the anterior subtalar joint capsule causes ligamentous laxity (Rose, 1991). CT scans show that the planus foot (hypermobile or flat foot) has no anterior sustentaculumtali facet (Smith, 1991). This suggests that this configuration is characteristic of a planus foot. Sustentaculumtali with only a medial facet has inadequate talar head support which allows excessive anterior and inferior rotation of the talus during weight bearing. This results in a valgus position of the calcaneus and a downward tilt of the talar head (Kapandji, 1970; Norkin, 1983). ${ }^{15-17}$

\section{CONCLUSION}

In our study Type $-\mathrm{C}$ was common, followed by Type - A. Race appears to play a part in the determination of the type of facets depict the marked differences in the talar facets on the calcanei between the various populations.

These morphological variations on the superior surface of the sustentaculumtali may predispose the people to joint instability, ligamentous laxity and the development of arthritic changes in the subtalar joint. People with the long continuous facet and only medial facet configurations of the sustentaculumtali may be at a greater risk for subtalar joint instability than individuals with the 2 - facet configuration.

The variations among talar articular sub types may be a consequence of external factors and also of anthropological factors which need further investigation.

\section{ACKNOWLEDGEMENTS}

We are thankful to Dr. Ratnabali Sen Gupta, Ex HOD and Professor Sikkim Manipal Institute of Medical Sciences, Sikkim for her help to provide the facilities to carry out the work.

\section{REFERENCES}

1. Ayoob A, Maeseneer MD, Shahabpour M, Van Roy P, Barbaix E and QIng S. The talocalcaneal unit: Pictorial review of anatomy and pathologic conditions on multi detector ct. JBR-BTR 2010; 93: 20-27. 
2. Mosier KM and Asher M.Tarsal coalitions andperoneal spastic flatfoot. Journal of Bone and Joint Surgery 1984; 7: 976-984.

3. Sharada R, Sneha K, Gupta C, Pai SR and Rairam GB. Non metrical study of the pattern of talar articular facets in south Indian dry calcanei. Surg Radiol Anat 2012; 34487-34491.

4. Gupta SC, Gupta CD and Arora AK. Pattern of talar articular facets in Indian Calcanei. J Anat 1977; 124:651-655.

5. Padmanabhan $R$. The talar facets of the calcaneus-an anatomical note. Anat- Anz 1986;161 (5): 389-392.

6. Bunning PSC and Barnett $\mathrm{CH}$. Variations in the talocalcanealarticulations. Journal of Anatomy 1963; 97:643.

7. Bunning PSC, Barnett $\mathrm{CH}$. A comparison of adult and foetaltalocalcanealarticulations. J Anat 1965; 99:71-76.

8. Eishi EH. Variations in talar articular facets in Egyptian Calcanei. Acta Anat 1974; 89:134-138.

9. Bunning PSC Some observations on the West African calcaneus \& the associated talocalcanealinterosseous ligamentous apparatus. American journal of physical Anthropology 1964; 22:467-472.

10. Campos FF and Pellico LG. Talar articular facets (faciesarticularestalares) in human calcanei. Acta Anat 1989; 134:124-127.

11. Leonard MA. The inheritance of tarsal coalition \& its relationship to spastic flat foot. A journal of bone and Joint Surgery 1974; 56b:520-526.

12. Olney BW and Asher MA. Excision of symptomatic coalition of middle facet of the talocalcaneal joint. The Journal of Bone and Joint Surgery 1987; 69-A: 539-544.

13. Rosenberg $Z$, Feldman $F$ and Singson RD. Intra-articular calcaneal fractures: Computed tomographic analysis. Skeletal Radiol 1987; 16(2):105-113.

14. Verhagen FD. Arthritis of the subtalar joint associated with sustentaculumtali facet configuration. J Anat 1993; 183:631-634.

15. Bruckner J. Variations in the human subtalar joint. Journal of Orthopedic and Sports Physical Therapy 1987; 8: 489-494.

16. Lee McGregor's synopsis of surgical Anatomy, GAG Decker publisher. 1986; ISBN0723608016. p. 543-544.

17. S. Standring. Gray's Anatomy, $40^{\text {th }}$ edition, Churchill Livingstone publisher. 2008; ISBN 9780443066849. p. 1437-1438.

\section{Authors Contribution:}

KA - Data collection, literature search and manuscript preparation; CP and BKT - Guided the project, data collection, analysis and interpretation; AK - Manuscript preparation and final approval of the manuscript.

Source of Support: Nil, Conflict of Interest: None declared. 